\title{
Carnets
}

Revue électronique d'études françaises de l'APEF

Première Série - 3 Numéro Spécial | 2011

La littérature face au "politiquement correct». Notions, pratiques et dérives

\section{Posture polémique de Richard Millet}

\section{Philippe Piedevache}

\section{(2) OpenEdition}

1 Journals

\section{Édition électronique}

URL : http://journals.openedition.org/carnets/6424

DOI : $10.4000 /$ carnets. 6424

ISSN : 1646-7698

Éditeur

APEF

Édition imprimée

Date de publication : 1 juin 2011

Pagination : 63-76

Référence électronique

Philippe Piedevache, "Posture polémique de Richard Millet », Carnets [En ligne], Première Série - 3 Numéro Spécial | 2011, mis en ligne le 19 juin 2018, consulté le 02 mai 2019. URL : http:// journals.openedition.org/carnets/6424; DOI : 10.4000/carnets.6424

\section{(c) (i) (9)}

Carnets est mis à disposition selon les termes de la licence Creative Commons - Atribution - Pas d'utilisation commerciale 4.0 International. 


\section{POSTURE POLÉMIQUE DE RICHARD MILLET}

PHILIPPE PIEDEVACHE

Université Lyon 2

philippe.piedevache@univ-lyon2.fr

\section{Résumé}

Richard Millet a été pendant de nombreuses années un écrivain confidentiel. Mais au tournant du siècle, il est devenu l'une des figures littéraires majeures du politiquement incorrect. Le but de cet article est de retracer son parcours et de montrer la corrélation entre l'évolution de sa position dans le champ littéraire (selon la terminologie définie par Bourdieu) et les transformations d'une parole qui aura gagné en liberté. Richard Millet a toujours été réticent devant le postmodernisme et les choix culturels et politiques de la France et ses premiers textes en témoignent, mais il s'agit de voir comment ses écrits ont pu être plus incisifs, plus combatifs à mesure qu'il étendait son pouvoir dans le monde des lettres. Dès lors, nous intégrons la question du politiquement incorrect dans le cadre plus large des stratégies de reconnaissance qui amènent l'un des plus écrivains les plus critiques sur son époque à participer à un jeu de rôles par lequel une position intellectuelle et posture médiatisée se rejoignent.

\section{Abstract}

For quite long, Richard Millet had a fairly narrow readership, but at the turn ot the century, he became one of the prominent literary figures of the politically correct. In this essay, I aim at tracing the artist's career and emphasizing the correlation between the evolution of his position in the literary field -as defined by Bourdieu- and the increasing freedom of his changing speech. As he can be seen from his first texts, Richard Millet has always had misgivings about postmodernism and the political and cultural orientations in France. My point here is to analyse to what extent the fighting spirit and incisiveness of his written work have grown in a parallel step with his influence in the literary world. The question of the acknowledgement strategies, which led a writer with one of the most critical eye of his time to take part in a rôle play uniting intellectual and mediatized positions.

Mots-clés: Richard Millet, politiquement correct, champ littéraire

Keywords: Richard Millet, politically correct, literary field 
Deux citations pour commencer:

Une langue sacrifiée à la paix civile, c'est la mort d'une culture millénaire. Je n'en rends nullement les immigrés responsables; les semeurs de vent, ce sont les idéalistes postchrétiens et les marchands d'esclaves au pouvoir. Les reliquats hystériques du gauchisme ont fait le reste: évacuer la dimension spirituelle de la culture. (Millet, 2008a: 39)

On ne parle plus de littérature mais de littératures: cela va de pair avec l'accession des déviances sexuelles au rang de normalité minoritaire. Devenue un "lieu" identitaire, la littérature est morte de sa pluralité relativiste, revendicatrice, expiatoire. (Ibid: 67).

Deux fragments de ce que Richard Millet écrit, entre autres, dans L'Opprobre, en 2008. Provocation? Volonté de rompre avec le politiquement correct? À voir. Au moins peutil l'écrire et le publier, et c'est dans la perspective de cette liberté acquise qu'il est intéressant d'étudier cet écrivain. Non pas tant pour discuter la validité de ses prises de positions politiques que pour essayer de décomposer la trajectoire d'un auteur à qui le champ littéraire autorise une telle échappée hors de ce que les conditions culturelles, intellectuelles et morales d'une société avaient fini depuis un certain temps par prescrire. II s'agit donc d'envisager le problème (si c'est un problème) d'une manière qui ressortit d'une question double: celle du possible (puis-je faire entendre ma voix?), celle de la protection (puis-je ne pas être étouffé par la machine du politiquement correct en essayant de faire entendre ma voix?). On comprendra déjà que l'approche que je fais du cas Millet renvoie d'une manière assez sensible à la théorie bourdieusienne du champ littéraire.

Richard Millet écrit depuis longtemps. Son œuvre est conséquente, étendue sur trois décennies. Il a été publié pour la première fois en 1983 et depuis, une trentaine d'ouvrages ont paru. Ce n'est donc pas un auteur rare, parcimonieux, économe de sa plume. S'il faut considérer cette aventure littéraire, mais plutôt devrais-je dire cette carrière dans l'univers des lettres, dans la sphère éditoriale et intellectuelle, on peut dégager trois périodes, dont je ne conteste pas qu'elles puissent avoir des limites parfois un peu floues. Mais elles ont, je pense, l'intérêt de marquer les temps d'une progression à la fois cohérente sur le fond et signifiante dans la forme.

La première, intitulons-la: le temps de l'artiste, recouvre une période allant de 1983 à 1993. L'écrivain est essentiellement publié par P.O.L. et ses ouvrages romanesques sont plutôt centrés sur une réflexion esthétique à travers laquelle les personnages s'interrogent sur leur place, leur rapport à la culture, et l'hiatus entre leurs aspirations et les conditions objectives d'une société dont ils sentent qu'elle est un frein à leur "réalisation". Ce qui 
résumerait en quelque sorte le Millet première version se trouve dans la trilogie: L'Angélus, La Chambre d'ivoire et L'Écrivain Sirieix, trois romans écrits entre 1988 et 1992, d'abord autonomes mais que l'édition Folio en 2001 a fini par réunir avec justesse dans un même volume. On y trouve déjà des éléments constitutifs d'une position intellectuelle s'inscrivant dans le décalage d'une société post-moderniste; on y repère un sérieux, une gravité qui trouvent leur réalité non tant dans le choix du personnage-artiste, mais dans les référents culturels mis en jeu. Le classicisme musical du narrateur de L'Angélus, lequel signale en passant qu'il lit Dans les jardins de Bérénice de Barrès, l'épigraphe de La Chambre d'ivoire tirée de L'Algèbre des valeurs morales de Marcel Jouhandeau, celle de L'Écrivain Sirieix, extraite cette fois du Fils de Louix XVI de Léon Bloy, ces simples exemples, mais fort symboliques, donnent, si j'ose dire, la coloration de l'écriture et des références. C'est l'invocation d'un classicisme direct ou indirect dont on sait qu'elle constitue l'un des signes identificatoires du réactionnaire depuis le XIXe siècle. Chacun des personnages est, sinon de Siom (le Viam natal de l'écrivain Millet), du moins du pays: la Corrèze. C'est pour cela d'ailleurs que l'auteur finit par confier dans la préface à l'édition Folio qu'on lira aisément ces textes comme "une ironique tentative d'autobiographie transposée" (Millet, 2001: 9), celle d'un homme qui a bien du mal à être de son temps, ce qui n'est pas une confidence sans importance puisqu'elle intervient au moment du virage polémique dont je parlerai ultérieurement. II faut donc considérer cette première période comme celle d'une expérience scripturale par laquelle Richard Millet utilise la figure classique de l'artiste comme paravent. Cette manière de procéder permet une relative neutralisation du discours auctorial. Le personnage n'est pas l'homme qui écrit et l'on ne peut, sous peine même d'être accusé d'un raccourci facile, de projeter l'un sur l'autre. En poussant même le paradoxe, il serait possible d'envisager cette figure de l'artiste, non selon un principe identificatoire, mais sous l'angle d'un contrat hyperbolique dont le lecteur ne doit pas être dupe. Et pour prendre une comparaison où cette fin de siècle en rejoint une autre: les artistes de Millet doivent être compris avec la précaution que l'on prendra pour ne pas calquer Des Esseintes sur Huysmans. II y a donc, à cette époque, une manière très littéraire pour l'écrivain d'avancer caché.

La deuxième période, intitulons-la: l'orientation corrézienne, dont je placerai les bornes entre 1993 et 2000, qui va se concentrer sur l'épopée siomoise. Avec, néanmoins, un point de départ qui mérite quelques éclaircissements, sans doute: la parution, dans sa forme complète, du Sentiment de la langue, en 1993 aux éditions de La Table Ronde. Ce texte me paraît être un nœud de l'œuvre milletienne. Les deux premiers tomes ont paru en 1986 et 1990, aux éditions du Champ Vallon. Ils prennent donc logiquement place dans la première période dont je viens de parler. Ils sont en quelque sorte le début d'une entrée de Millet dans la polémique et fera-t-on remarquer un démenti à mon découpage temporel. II n'y pas 
pourtant à mon sens nulle contradiction. Dans la préface qu'il lui consacre, Yannick Haenel rappelle que ce texte se réfère à un genre ancien: le mélange, et il en définit l'usage, là encore, suranné dans un monde où les individus vont se coucher juste après avoir éteint la télévision: "le livre de chevet" (Millet, 1993: 9). Son objet est moins la polémique que le partage, moins la vindicte que la promenade, moins le combat (même si la rudesse n'est pas exempte de ces pages) que la mélancolie. Et le troisième tome publié en 1993, qui lui donne sa forme achevée, n'y change rien. Cette publication est donc marquée, si on la compare à ce que sera la troisième période de Millet, par une retenue dont il est certain qu'elle est aussi conditionnée par la situation institutionnelle de l'auteur à cette époque, au moment de la publication et non lorsque le livre est reçu par le public, c'est-à-dire quand l'écriture ne peut encore prévoir son destin. Mais, en 1994, cet ouvrage obtient le Prix de l'Essai de l'Académie française, et grâce à lui, Millet une reconnaissance du monde littéraire, et non des moindres. II n'est donc plus un auteur inconnu ou très confidentiel. C'est dans ces temps qu'il publie deux romans majeurs, denses, âpres, centrés sur la Corrèze: La Gloire des Pythre, L'Amour des trois sœurs Piale. Par la peinture d'un univers en déliquescence, en centrant sa matière romanesque sur la destruction progressive d'un monde à la fois abandonné de la modernité ultime et réduit à n'être qu'un rebut de cette même modernité ultime, Millet approfondit son exploration d'un divorce entre un passé représenté par le pays historique, ancré dans la terre, et un univers contemporain, lointain et inquiétant. Revendiquant, lui, le lointain intérieur et l'épopée immobile, l'écrivain continue à se démarquer, dans la matière littéraire choisie, d'une littérature française encore centrée sur le récit auto-fictionnel, le nombrilisme germanopratin. II y fustige, dans le cadre d'une narration somme toute assez classique, dont le phrasé obéit souvent à des réminiscences proustiennes (et l'on comprend le lien avec ses propos développés dans Le Sentiment de la langue, justement), le désordre d'un monde qui oublie de s'inscrire dans le temps. Prenant la forme d'un hommage à un monde perdu, c'est-à-dire considérant le point le plus éloigné dans le temps (et symboliquement dans l'espace, au regard d'une distinction -avec un écho de l'acception bourdieusienne- entre Paris et la province, la ville et la campagne), l'écrivain prend la défense de ceux que l'élan du progrès a sacrifiés. La narration abandonne les artiste pour se tourner vers les petits, les médiocres, les gens de peu, ce qui jusqu'alors était plutôt l'apanage de la littérature dite de terroir. Ce changement n'est pas tant une question de milieux (et je ne l'entends pas selon l'acception qu'en donnaient notamment les naturalistes) qu'une évolution politique. En effet, c'est au cœur de la Corrèze que Millet rend lisible son idéologie littéraro-politique (ou éthico-politique, si l'on veut prendre le versant spirituel). Et il ne cesse de ré-injecter dans son texte des considérations qui renvoient, elles, à des problématiques contemporaines, sur les vaincus de l'histoire (et c'est bien ainsi que sont présentés les personnages principaux, et ces vaincus sont des autochtones français), sur la 
question de la langue comme question morale et politique. Et, dès lors, on ne s'étonne plus des remarques récurrentes sur le français et le patois dans La Gloire des Pythre; on prendra comme symbolique le début de L'Amour des sœurs Piale essentiellement tourné sur cette problématique. Je renvoie aux quatre premières pages. Elles sont éloquentes. Cette même imposition de la question linguistique (encore que l'adjectif nous semble trop technique au regard des implications affectives, quasi charnelles que Millet suppose dans la relation à/de la langue) inaugure Ma Vie parmi les ombres:

Après moi la langue ne sera plus tout à fait la même. Elle entrera dans une nuit remuante. Elle se confondra avec le bruit d'une terre désormais sans légendes. Les langues s'oublient plus vite que les morts (Millet, 2003: 15)

Cette œuvre appartient à la troisième période mais il est clair qu'on y retrouve, on y entend pleinement les échos de l'essai primé. Cette problématique est sans nul doute le sillon transcendant de l'entreprise milletienne, le terreau de son écriture, la matière de sa terreur.

Ce qui est intéressant, je crois, c'est de voir comment l'irruption du présent se fait dans une sorte de construction à rebours. Le présent est, d'une certaine manière, l'élément qui laisse sa trace dans le récit. II n'est pas le sujet mais il est, du point de vue de l'auteur, une situation terminale à partir de laquelle tout peut se construire. II faut ainsi comprendre qu'à ce moment-là, Millet encorsète une position déjà repérable antérieurement dans le cadre strictement littéraire d'une narration qui se pose comme fiction relativement nostalgique, en limitant ainsi les effets éventuellement polémiques. Son évocation d'un territoire désormais mort (le Viam réel devenu Siom fictif) modalise et donc freine la tension violente entre le passé et le présent. Le recul (ou le retrait) permis par la délégation à une voix narrative qui n'est pas l'auteur préserve le texte de la tentation d'une vindicte trop forte, trop épidermique. Certes, c'est l'occasion, pour une certaine presse, de moquer ce provincialisme, mais l'expérience milletienne demeure, dans l'ordre de l'écriture, celle d'une formalisation avant tout romanesque. II serait alors difficile de lui faire un procès politique, de le réduire à un romancier réactionnaire intégral.

Troisième période, intitulons-la: le virage polémique, à partir des années 2000 , jusqu'à aujourd'hui. L'élément de rupture est, me semble-t-il, la parution de Lauve le pur. Ce roman de la désillusion violente d'un enseignant en banlieue signe une inflexion radicale (ceci dit sans aucun jugement de valeur) de l'écrivain par rapport à son discours antérieur. II y est certes encore question de Siom, à travers le contrepoint de cette voix chorale à laquelle, d'une certaine manière, se confie le héros, mais le changement est très sensible. II ne s'agit plus seulement d'évoquer le désastre d'un monde perdu dans le passé; il faut 
encore envisager la déliquescence présente à travers une remise en cause plutôt abrupte de certaines valeurs du politiquement correct. C'est par le biais d'un regard acide sur le monde enseignant, sur ses engagements et surtout sur ses renoncements que Millet prend le virage clair de la polémique. Pour ce faire, l'écrivain alimente la fiction de son expérience de professeur. Le cadre fictionnel est comme forcé par le redoublement d'une voix narrative investie par l'auteur. L'ambition est alors assez identifiable: en finir avec la mythologie républicaine de l'école comme lieu de culture et de formation du citoyen, et avec cette mythologie, rompre avec l'idéologie indiscutable de l'éducation des masses. Ainsi en est-il de l'enseignement public: "cimetière de l'esprit", "refuge des solitaires et en particulier des femmes, celles qui n'en pouvaient plus de se morfondre chez elles" (Millet, 2000: 247), décrire avec sévérité ce qui n'est plus "un métier digne de ce nom mais un combat perdu d'avance" (Ibid: 114). On cerne alors nettement comment l'écrivain durcit son discours. II ne s'agit plus de s'en tenir à un monde ancien, perdu, à une quête impossible sur l'axe inversé de la temporalité. Le cœur du récit n'est plus le disparu/la disparition mais ce qui est en train de se déliter, et à travers cette observation la dénonciation d'une hypocrisie que l'on ne peut circonscrire au seul domaine des mensonges de l'opportunisme politique mais qui se révèle comme signe d'un abandon, d'un renoncement moral et culturel. L'attaque change de registre et elle œuvre effectivement dans le présent. Le fait même qu'une partie du récit se déroule en banlieue et à Paris est significatif. En rabattant le cadre temporel (le présent) sur un nouveau cadre spatial (la banlieue), Millet s'invite au débat contemporain autour du futur de la culture européenne. Ce durcissement se retrouvera dans la somme siomoise qu'est $\mathrm{Ma}$ Vie parmi les ombres, publiée en 2003, dont le titre est évidemment à analyser dans sa double composante: les ombres du passé et celles, plus mortifères, d'un présent où la France n'est plus que l'ombre d'elle-même. Somme siomoise avec des accents apocalyptiques qui est la dernière marche romanesque avant tout ce qui tourne autour de la polémique franche: Harcèlement littéraire, Le Dernier Écrivain, Désenchantement de la littérature, L'Opprobre.

La composition de ces trois périodes, aussi schématique soit-elle, met en perspective une constance milletienne dans la posture non consensuelle face à une accréditation libérale franche et aux critiques d'une gauche qui n'en marque pas moins, malgré tout, sa confiance en une évolution progressiste de la société (post)moderne. La trame de sa pensée est solide et Millet s'inscrit dans une tradition antimoderne, si l'on veut reprendre la catégorisation de Compagnon. Chantal Lapeyre-Desmaison approche sous cet angle son œuvre dans une communication lors du colloque d'Arras qui lui est consacré. Néanmoins ce n'est pas sur ce noyau idéologique qu'il faut nous arrêter; il s'agit plutôt de recouper le trajet de cette tension visible, tension de plus en plus forte, avec les conditions d'écriture et l'inscription de l'auteur dans le champ littéraire. Or, celles-ci ont considérablement changé au cours des presque 
trente années de littérature. II y a sur ce point deux grands moments éditoriaux.

De 1983 à 2000, Millet est publié par P.O.L., maison reconnue, avec des choix d'ouvrages voulant marquer comme on dit une différence, peut-être pas sur le mode radical de l'écriture blanche de Minuit, mais n'ayant pas forcément le souci d'une lecture immédiate, facile. Elle ose découvrir des talents, mise sur des auteurs parfois alternatifs (il suffit de voir ce qui est publié en poésie, par exemple). En retour, il est clair que sur le plan de la pénétration auprès du lectorat, P.O.L. reste relativement en retrait. L'estime et la valorisation élitiste priment sur la reconnaissance purement commerciale. II n'est donc pas étonnant que Richard Millet soit à cette époque un écrivain confidentiel, dont la voix ne peut guère porter. Qui plus est, nous l'avons signalé, le choix de ses thématiques (autour de l'artiste notamment) ne peut que le maintenir dans ce retrait, à la fois sur le plan esthétique et institutionnel. Retrait dont il sort certes à partir de l'année 1994 par le biais de la reconnaissance académique déjà évoquée, et qui se confirme par le succès des livres siomois: La Gloire des Pythre et L'Amour des trois sœurs Piale. Succès dont on ne dira nullement qu'il est le fait d'une soudaine concession au lectorat mais qui néanmoins peut être éclairé par l'évolution du contexte politique et culturel français. Sur le plan littéraire, on soulignera que le retour de la province s'amorce, que certains auteurs la prennent en héritage, pour reprendre le titre d'un livre de Sylviane Coyault, et non des moindres: Pierre Bergounioux ou Pierre Michon.

Cette réussite littéraire permet clairement à Richard Millet de pouvoir franchir un seuil, d'occuper une autre position dans le champ littéraire, puisque l'enseignant en banlieue qu'il était jusqu'alors entre de plain-pied dans le monde de l'édition en 1998, d'abord chez Balland (pour la collection grise) puis, et c'est là une modification de taille, chez Gallimard où il intègre le comité de lecture. Le saut est disons-le qualitatif, dans le sens où, par sa fonction, Millet acquiert une puissance symbolique et effective, et il apparaît de facto un personnage important de la République des Lettres. Ce changement de statut social a aussi une incidence dans son trajet propre d'écrivain, puisqu'après Lauve le pur (2000), il devient luimême un auteur Gallimard. II est de plus en plus identifiable et d'une façon certaine sa marge de manœuvre devient plus grande. Il est d'ailleurs notable que ses publications s'accélèrent. II a une position. Et si les recensions et les questions de statistiques ne sont pas tout, remarquons simplement: les entretiens qu'il accorde (ou qu'on lui demande?) sont fort limités jusqu'en 2001: six, puis beaucoup plus fréquentes ensuite: 12 entre 2001 et 2005. Il devient un sujet universitaire (la thèse de Sylviane Coyault parue en 2002, colloque de I'Université d'Artois en 2006, colloque auquel il se rend d'ailleurs). Celle-ci est le fruit d'un travail littéraire indéniable, d'une opportunité qui lui a été offerte (sans qu'il faille parler d'opportunisme) et d'une conjoncture éditoriale très rapidement favorable, puisqu'il est I'homme qui appuie la publication des Bienveillantes de Jonathan Littell, dont le succès, dans 
les contraintes économiques fortes imposées aux éditeurs, ne peut qu'affermir son statut. Cette nouvelle situation a des incidences sensibles quant à sa latitude dans la sphère littéraire. Lui qui fut longtemps à la périphérie du pouvoir est désormais au cœur du pouvoir.

Dans un article paru dans Lire, en mai 2008, au sujet du si controversé L'Opprobre, le journaliste Baptiste Liger signale qu'il ne lui a pas été possible de trouver un interlocuteur dans le petit monde des Lettres pour apporter la contradiction à l'auteur. II fait donc peur. Liger, dans cet article, rapporte que Jérôme Garcin, homme d'influence s'il en est, ne s'étonne pas de cette frilosité à affronter Millet: c'est là le signe de sa puissance acquise en quelques années. D'ailleurs, dans un numéro du Magazine littéraire, en décembre 2007, quelque six mois auparavant, on trouvait un entretien entre "deux écrivains qu'on ne présente plus" (ainsi écrit la journaliste): Philippe Sollers et Richard Millet. Rencontre dont on attend au demeurant qu'elle soit explosive, puisque Millet ne manquera pas d'épingler Sollers dans L'Opprobre, justement, qui paraît en mars 2008. Mais, comme ce qui souvent arrive dans une société du spectacle permanent où l'effet d'annonce est l'essentiel, l'entretien est décevant. S'ils ne sont pas du même avis, les deux hommes évitent les charges. L'ensemble reste très courtois, et somme toute convenu. Plus encore: Philippe Sollers trouve qu'à l'endroit de Millet, il y a eu une "réaction inquisitoriale, et [...] même stalinienne" (Millet, 2007b). Ce commentaire est intéressant en ce qu'il induit que l'homme du cosmopolitisme mondain, forteresse littéraire d'un certain politiquement correct, adoube par là même celui qui lui est contraire. Trois ans auparavant, pour l'hebdomadaire L'Express, Millet avait déjà accepté de débattre avec une autre figure très médiatique: Frédéric Beigbeder. Ces face-à-face, dont l'intérêt est très relatif me semble entrer dans un processus d'identification/authentification de cet écrivain comme la figure emblématique du politiquement incorrect. Cette mise en scène de soi peut surprendre de la part d'un auteur qui se targue d'être un solitaire, passablement misanthrope, mais elle procède assez logiquement d'un élan narcissique très postmoderne. Et, en effet, la troisième période que j'ai définie plus haut correspond à un recentrage très net de Millet autour de sa personne. La polémique dans laquelle il s'installe enfle à mesure que son institutionnalisation prend de l'ampleur et, très clairement, Harcèlement littéraire, Le Dernier Écrivain, Désenchantement de la littérature et L'Opprobre voient un glissement sensible du discours-scandale à l'êtrescandale, c'est-à-dire celui qui est scandaleux mais aussi celui qui postule le scandale comme caractéristique de soi.

Cela fonctionne d'ailleurs admirablement. Il devient même une matière journalistique de choix dans le registre de l'agressivité. On lui reproche ici d'être une "moulinette à clichés", là d'“enfoncer des portes ouvertes" (Savigneau), ailleurs de "déraper" voire de faire "un pétage de plomb" (Liger). On se retrouve ainsi devant une sorte de paradoxe qui voit s'installer dans le champ littéraire français, et à une place forte (dans Le Nouvel Observateur 
du 27 mars 2008, Jérôme Garcin titre ainsi son billet: Richard Millet 1er), l'antimoderne, le réactionnaire, pour user d'un vocabulaire où se mélangeraient les traits culturels et politiques. On peut donc dire que Millet a réussi à contourner brillamment l'écueil du politiquement correct, c'est-à-dire le poids de la représentation collective, notamment dans la sphère intellectuelle, le déséquilibre entre celui qui veut enfreindre la règle et les gardiens d'une orthodoxie morale. II devient une sorte de mètre-étalon d'une contestation politicolittéraire et c'est à lui que l'on vient demander tout à coup les oracles autour d'une déchéance culturelle annoncée. C'est en ce sens qu'il faut comprendre, par exemple, l'entretien qu'il accorde à Jacques-Pierre Amette pour Le Point en 2007. Cela signifie que son activité éditoriale propre (comme écrivain) se double dès lors d'une présence médiatisée donnant l'impression d'une toute puissance somme toute assez singulière. Et c'est d'ailleurs dans cette perspective qu'il faut entendre sa réponse à une question d'Amette qui propose un parallèle entre les romantiques et lui:

Vous voulez absolument désamorcer ce que j'écris? Sur le romantisme, je pencherai plutôt vers Chateaubriand. Non pas que je me compare à lui! Les Mémoires d'outretombe dévoilent quelqu'un qui a la conscience qu'il passe d'un monde à un autre. C'est le leitmotiv des Mémoires, je viens de les relire. Cela m'a frappé, ce passage. Je suis né dans un ancien monde, je suis dans un nouveau monde... Que puis-je faire à partir de ça? La filiation, c'est important. Mais essayez de voir quelqu'un qui a une filiation aujourd'hui... Beigbeder, qui se revendique de Scott Fitzgerald. Autre chose: je pense que le fait de vivre dans cette espèce d'Europe est mortellement ennuyeux. On est sortis de l'Histoire. (Millet, 2008b)

Il y a, dans l'initiale question rhétorique, puis dans les références invoquées, une manière d'imposer sa figure comme celle de la résistance et l'allusion, évidemment dépréciative, à Frédéric Beigbeder prête à sourire. On remarque là une sorte de va-et-vient qui prend son sens justement dans des interactions liées à des enjeux de pouvoir en grande partie issus de l'intégration de la sphère littéraire dans l'espace médiatique.

II serait absurde de mettre cela sur le compte d'un machiavélisme carriériste, et aussi grand soit l'ego de Richard Millet, il ne faut pas interpréter sa littérature comme un simple jeu d'ambition. Si l'écriture n'est pas dépourvue de rêves dorés (le côté Frédéric Moreau...) dans le monde social, elle est d'abord, chez lui, une question d'esthétique et il faut reconnaître à Millet la beauté de son écriture. Néanmoins, on reprendra encore une fois la trajectoire milletienne, sur le fond, pour remettre en perspective sa posture de politiquement incorrect et en nuancer l'originalité.

Lui-même l'avoue dans un entretien: "l'époque veut que, pour pouvoir être entendu, il faut exagérer. Nous vivons sous la dictature du politiquement correct, qui refuse un autre réel 
que le sien" (Liger). Deux ouvrages mettent particulièrement en pratique ce constat: Le Dernier Écrivain, paru en 2005 et Désenchantement de la littérature, publié trois ans plus tard. Un petit commentaire sur les titres est je crois nécessaire. Ils dramatisent d'emblée le propos, le placent dans une perspective radicale de perte, d'abandon: posture classique de l'antimoderne. Ils biaisent aussi singulièrement l'idée que l'on peut se faire de ces opuscules (ce sont des textes brefs) car il n'est pas, loin s'en faut, question de la seule littérature. La place de I'histoire, la faillite de la langue, la non-transmission du savoir, l'appauvrissement intellectuel du monde contemporain, sa désagrégation spirituelle, le renoncement de l'Europe face à l'Islam,... le programme est varié. Est-ce un simple hasard, une sorte de maladresse, d'approximation? Considérons plutôt qu'en titrant ainsi ce qui constitue la partie pamphlétaire de son œuvre, Millet oblige le lecteur à appréhender son politiquement incorrect non sous l'angle d'un engagement politique déterminé, mais comme l'expression d'un écrivain libre, renouant ainsi une tradition française qui avait été mise en veilleuse depuis longtemps. C'est donc sous le jour d'une filiation esthétique que l'auteur, cette fois, se pare contre toute assimilation (et donc réduction) à un territoire intellectuel identifié. II est, à lui seul, une entité irréductible au politiquement correct. Et comme cette solitude a besoin d'étais solides, Millet choisit alors le pamphlet, la parole dure, radicale, une sorte de réactivation de la violence littéraire que l'on trouvait jadis chez Bloy ou Bernanos. Ce sont ces auteurs-là qui s'inscrivent en filigrane de l'écriture de ces deux textes. La voix milletienne procède d'une logique où la parole (tout autant que le propos lui-même) est réaction, là où remontent au plus visible le parti pris des choses, les formes structurées de l'idéologie. La vocifération et le déluge verbal sont des armes classiques. Encore faut-il pouvoir (non pas seulement techniquement mais légitimement) les employer. Or, ce politiquement incorrect qui, tout à coup, enrage, l'écrivain le donne tel à partir du moment où les moyens institutionnels (et le champ littéraire est une institution; il a ses féodalités) sont de son côté. On prête plus facilement à ceux qui ont déjà.

II est d'ailleurs très intéressant de faire le rapprochement suivant: une fois ce détour, ce dis-cursus, pamphlétaire accompli, Millet revient à une forme moins XIXe siècle, celle de l'écriture fragmentaire. L'Opprobre, qui est une réponse au procès qu'on lui a fait pour $L e$ Désenchantement de la littérature, reprend, malgré la violence de certains propos (comme un écho bloyen), la structure choisie pour Le Sentiment de la langue. Coincidence? Ou bien le signe que justement une forme plus classique (un peu XVIle en somme, un côté La Rochefoucauld) marque mieux le discours de la contestation devant le rouleau compresseur d'un système dénoncé par l'auteur? À moins que ce ne soit une démonstration de maîtrise à l'endroit de ceux qui usent désormais envers lui de l'invective et qui en font de facto un martyr du politiquement correct? En effet, si pour reprendre Jérôme Garcin, Millet peut se prendre pour le roi, il est aussi, pour certains, Richard M, le Maudit, ainsi que titrent Franz- 
Olivier Giesbert et Christophe Ono-dit-Biot, pour un entretien publié dans Le Point en janvier 2009. Le même hebdomadaire revient sur cette thématique en novembre 2010 pour un nouvel entretien où se croisent cette fois les voix de Millet et de Marc-Édouard Nabe: Les maudits parlent.

Ne serait-ce pas une stratégie pour discréditer le politiquement correct que de faire la démonstration de son terrorisme? Et de se poser soi en victime, lui qui affirme dans cet entretien de 2010 que l'on

veut [sa] mort économique, ne pouvant [l]'atteindre symboliquement: [ses] derniers livres sont boycottés par la plupart des journaux, notamment les suppléments littéraires de la presse socialo-gaucho-petite-bourgeoise: un vrai "sans-papiers", un non-citoyen de l'empire du bien (Millet, 2010).

De fait, l'écriture de Millet tend vers une exposition certes narcissique mais aussi dramatisée pour asseoir l'idée qu'il lutte contre vents et marées pour se faire entendre, pour qu'on ne l'écrase pas. L'Opprobre est sous-titré ainsi: essai de démonologie. II s'agit bien d'un procès en sorcellerie dont l'auteur se croit l'objet. Pour quelle raison? Lui-même s'en explique dans ce même entretien: "J'écris: je m'affronte au réel, je dis la vérité, plutôt que de devenir un sociologue de l'immédiateté, un thuriféraire du présent absolu, un théologien de l'autoaveuglement..." (Ibid).

Cette définition par la négative, par ce qu'il n'est pas, par ce qu'il refuse d'être, a son corrélat, dans une affirmation qu'il juge évidemment provocatrice. II est "le minoritaire même: blanc, mâle, 'Français de souche', catholique, hétérosexuel” (Millet: 2005b, 28), retournant ainsi avec une certaine jubilation le concept deleuzien de déterritorialisation tel qu'on le trouve dans le Kafka. Pour une littérature mineure publié en 1975. Ce discrédit recherché sera d'autant plus envisageable que Millet a pour lui une singularité biographique, d'être l'homme de deux cultures, de faire coexister en lui ce qu'il appelle sa "francité" et sa "libanité". Millet en joue d'ailleurs, lorsqu'il reprend, dans L'Opprobre, les caractéristiques revendiquées de cette auto-définition.

Provincial, catholique, Blanc, hétérosexuel et recherchant la pureté en toute chose: parfait métèque, Levantin, même, avec quelque chose de la grande cruauté asiatique et de son indifférence à l'homme. (Millet, 2008a: 75)

II ne s'agit pas de mettre en doute son attachement à l'Orient, au Liban, en particulier, mais il est évident que les deux composantes de la définition, en se combinant, tendent à désamorcer la radicalité de la première partie (radicalité entendue évidemment au regard du 
politiquement correct). Plus encore, elles neutralisent l'attaque classique contre les réactionnaires auxquels Millet est assimilé: le repli identitaire occidental aux relents racistes. Il y a donc bien, dans la tentation de l'Orient avec laquelle l'écrivain travaille son image, une manière de rendre la vie impossible à ceux qui veulent sans cesse lui faire des procès d'intentions. En singularisant sa position (et il est indéniable qu'elle est singulière, ne seraitce que par les éléments biographiques qui en sont le terreau), Millet gagne un surcroît de légitimité dans la critique de la morale autorisée par le politiquement correct.

Mais, cet engagement dans la critique morale et politique, n'est-il pas, lui aussi, un trompe-l'œil? Dans un article sur la Colère de Richard Millet, Bruno Chaouat parle d'un complexe d'Alceste (Chaouat, 233) qui traverserait l'esprit d'un certain nombre de mécontemporains: Finkielkraut (et l'on pense évidemment à La Défaite de la pensée datant de 1986), Jean-Claude Michéa, Philippe Muray... Le retour en grâce, dans le monde littéraire, de certains auteurs honnis: Bernanos, Jouhandeau, Drieu la Rochelle (qui va enfin entrer à la Pléiade) participe d'un retour de balancier contre ce qui fut la domination de gauche de l'après-guerre dans l'espace culturel. Dès lors, le politiquement incorrect aurait pris doucement ses quartiers dans une société française en crise. Crise politique, crise culturelle, crise identitaire, crise sociale... Un certain nombre de thématiques du discours contestataire de Millet ont désormais voix au chapitre, y compris dans les allées de l'État et dans les discours gouvernementaux ou d'opposition. La droitisation des idées, le raidissement idéologique sont des faits sensibles, et qui sont apparus au milieu des années 80. Ce qui longtemps fut tu s'exprime maintenant. Les attaques sur le déclin politique et culturel de la France, la remise en cause d'un modèle cosmopolite qui vire doucement vers le repli identitaire, le procès des bonnes consciences de gauche en matière sociale: tous ces phénomènes ont commencé d'imprégner la société française, ce que d'aucuns ont, par exemple, considéré sous la dénomination un peu simpliste de lepénisation des esprits. II ne s'agit nullement d'assimiler Millet à un écrivain d'extrême-droite. Surtout pas. Sur ce plan, sa posture est beaucoup plus retorse à l'analyse, empruntant à bien des traditions en apparence antagonistes. On trouverait chez lui des éléments passablement libertaires. Mais sa défiance vis-à-vis de la démocratie est un lieu commun de la pensée politique et Emmanuel Todd le rappelle avec beaucoup de justesse dans Après la démocratie. II est donc évident que son discours ne peut qu'être que mieux accueilli, ou toléré, aujourd'hui, surtout si, à côté des imprécations contre une société décadente, l'écrivain déploie une œuvre complexe, protéiforme, soucieuse du style et paradoxalement en recherche d'une réconciliation entre l'Orient et l'Occident (comme un défi ultime à ceux qui voudraient le placer sur l'échiquier du choc des civilisations).

Dès lors, la question d'un Millet radicalement à contre-courant est peut-être posée, et de nous retrouver, au delà de son cas particulier, devant une situation fort embarrassante: ce 
ne serait pas tant la disparition d'un politiquement incorrect défini comme un interdit qui nous arrête (car il n'est pas inconcevable que tout ne puisse pas être acceptable) mais sa neutralisation et sa récupération comme posture intégrée au système de la communication généralisée, foisonnante, et, qui sait?, étouffée dans la masse, caractéristique de l'époque contemporaine. Lorsqu'il a l'impression de précher dans le désert, de convenir que sa voix est de celles qui vont se taire tout en ayant acquis le droit de s'exprimer, Millet ouvre peutêtre, et malgré lui, le débat sur une problématique plus terrible. Dans un monde voué à l'économique et à la financiarisation, les oppositions idéologiques ne sont que des leurres et ce qui guette (mais cela n'a-t-il pas déjà commencé?), c'est la disparition sensible de l'écrivain comme voix qui porte. 


\section{Bibliographie}

BOURDIEU, Pierre (1992). Les Règles de l'art. Paris: Seuil, coll. "Points"

ChAOUAT, Bruno. (2010). "Colère de Richard Millet". In: Boyer-Weinmann, Martine, Martin, Jean-Pierre (org). Colère d'écrivains. Nantes, Cécile Defaut.

Compagnon, Antoine. (2005). Les Antimodernes. De Joseph de Maistre à Roland Barthes. Paris: Gallimard, "Collection Bibliothèque des idées".

COYAULT, Sylviane (2002). La Province en héritage. Pierre Michon, Pierre Bergounioux, Richard Millet. Genève: Droz.

Deleuze, Gilles et GuatTARI, Félix. (1975). Kafka. Pour une littérature mineure. Paris: Minuit.

LAPEYRE-DeSMAISON, Chantal, (2008). "L'espace romanesque de Richard Millet: un manifeste antimoderne". In: Morzewski, Christian (org). Richard Millet: la langue du roman. Arras. Artois Presses Université. pp. 17-31.

MiLlet, Richard.(1993). Le Sentiment de la langue, I, II, III. Paris: La Table Ronde, "collection le Petit Vermillon".

MILLET, Richard.(1995, éd. revue 2003). La Gloire des Pythre. Paris: Gallimard, "Folio" en 1997

MILLET, Richard.(1997). L'Amour des trois sœurs Piale. Paris: Gallimard, "Folio" en 1999

MILLET, Richard.(2000). Lauve le pur. Paris: Gallimard, “Folio" en 2001

MilLET, Richard.(2001). L'Angélus (1988). La Tour d'ivoire (1989). L'écrivain Sirieix (1992). Paris: Gallimard, "Folio".

Millet, Richard.(2003). Ma Vie parmi les ombres. Paris: Gallimard, "Folio" en 2005.

MILLET, Richard.(2004), Fenêtre au crépuscule (conversation avec Chantal Lapeyre-Desmaison). Paris: La Table Ronde.

MILLET, Richard.(2005a). Harcèlement littéraire (entretiens avec Delphine Descaves et Thierry Cécille). Paris: Gallimard.

MILLET, Richard.(2005b). Le Dernier Écrivain, Paris: Fata Morgana.

Millet, Richard, Beigbeder, Frédéric(2005c). “Entretien”, L'Express, numéro 19, du 23 mai 2005.

MILLET, Richard.(2007a), Désenchantement de la littérature. Paris: Gallimard.

MILLET, Richard, SOlLeRS, Philippe (2007b), "Entretien", Le Magazine littéraire, numéro 470, du 12 décembre 2007.

MILLET, Richard. (2008a). L'Opprobre. Essai de démonologie. Paris: Gallimard.

MILLET, Richard. (2008b). "Entretien" (avec Pierre-Jacques Amette), Le Point, numéro 1826, du 30 août 2008.

MILLET, Richard. (2009). "Entretien" (avec Franz-Olivier Giesbert et Christophe Ono-dit-Biot), Le Point, numéro 1897, du 22 janvier 2009.

MILLET, Richard, NABE, Marc-Édouard. (2010) "Entretien”, Le Point, numéro 1992, du 18 novembre 2010.

LIGER, Jean-baptiste, "Le masque du démon”, Lire, numéro 365, mai 2008.

SAVIGNEAU, Jocelyne, "Quand Richard Millet se proclame "contemporain capital", Le Monde des Livres, 1er juillet 2005.

TODD, Emmanuel (2008). Après la démocratie. Paris: Gallimard, "Folio" en 2010. 